\title{
T Cell Receptor Fused to CD3 $\zeta$ : Transmembrane Domain of CD3 $\zeta$ Prevents TCR Mis-Pairing, Whereas Complete CD3 $\zeta$ Directs Functional TCR Expression
}

\author{
Coen Govers ${ }^{1}$, Zsolt Sebestyén ${ }^{1}$, Cor Berrevoets ${ }^{1}$, Hanka Venselaar ${ }^{2}$ and Reno Debets ${ }^{1, *}$ \\ ${ }^{1}$ Laboratory of Experimental Tumor Immunology, Dept. Medical Oncology, Erasmus MC-Daniel den Hoed Cancer \\ Center, Rotterdam, The Netherlands \\ ${ }^{2}$ Center for Molecular and Biomolecular Informatics, Nijmegen Center for Molecular Life Sciences, Radboud University \\ Nijmegen Medical Center, The Netherlands
}

\begin{abstract}
TCR gene therapy represents a feasible and promising treatment for patients with cancer and virus infections. Currently, this treatment rationale is hampered by diluted surface expression of the TCR transgene and generation of potentially self reactive T-cells, both a direct consequence of mis-pairing with endogenous TCR chains. As we reported previously (Gene Ther 16:1369, 2000; J Immunol 180:7736, 2008), TCR mis-pairing can be successfully addressed by a

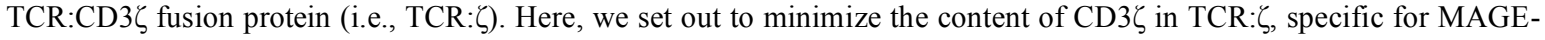
A1/HLA-A1, without compromising TCR pairing and function. Domain-exchange and 3D-modeling strategies defined a set of minimal TCR: $\zeta$ variants, which, together with a murinized and cysteine-modified TCR (TCR:mu+cys), were tested for functional TCR expression and TCR pairing. Our data with Jurkat T cells show that the CD3 $\zeta$ transmembrane domain

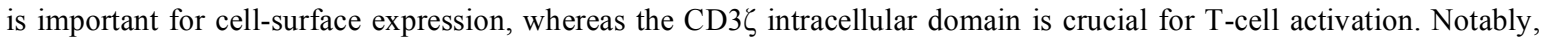
inability of TCR: $\zeta$ to mis-pair was not observed for TCR:mu+cys, which depended exclusively on the transmembrane domain of $\mathrm{CD} 3 \zeta$ and could not be recapitulated by a limited number of structurally defined $\mathrm{CD} 3 \zeta$ transmembrane amino

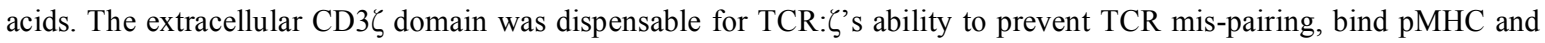
mediate NFAT activation. In primary human T cells, however, minimal TCR: $\zeta$ without CD3 $\zeta$ 's extracellular domain but not TCR: $\zeta$ nor TCR:mu+cys revealed compromised cell surface expression and T cell function. Taken together, our study demonstrates that $\mathrm{CD} 3 \zeta$ 's transmembrane domain dictates TCR: $\zeta$ 's inability to TCR mis-pair, but only TCR coupled to complete $\mathrm{CD} 3 \zeta$ and not its minimal variants were functionally expressed in primary $\mathrm{T}$ cells.
\end{abstract}

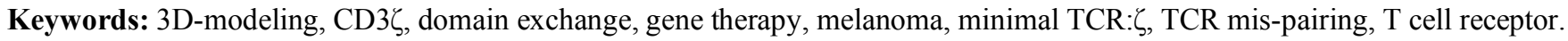

\section{INTRODUCTION}

$\mathrm{T}$ cell receptor (TCR) gene therapy is based on retroviral transduction of $\mathrm{T}$ cells with tumor or virus-specific TCR $\alpha \beta$ transgenes. Clinical TCR gene therapy trials proved feasible and applicable toward multiple tumor types, such as metastatic melanoma, colorectal carcinoma and synovial sarcoma [1-4]. This first series of studies with TCR-engineered T cells, although showing variable clinical responses in limited numbers of patients, demonstrates that responses generally lag behind those observed with non gene-engineered $\mathrm{T}$ cells [5-9]. In addition, in case of TCRs directed against MART-1, gp100 or CEA, but not the cancer testis antigen NY-ESO-1, treatment resulted in on-target toxicity, i.e., severe inflammation of healthy tissue expressing the target antigen $[1,2,4]$.

The efficacy and safety of clinical TCR gene transfer may be further enhanced by strategies that address TCR

*Address correspondence to this author at the Groene Hilledijk 301, 3075EA Rotteram, Laboratory of Experimental Tumor Immunology, Dept. Medical Oncology, Erasmus MC-Daniel den Hoed Cancer Center; Tel: +31 10 7041718; Fax: +31 10 4071005;

E-mail: j.debets@erasmusmc.nl mis-pairing. TCR mis-pairing is a recognized phenomenon of TCR gene transfer, and defined by the incorrect pairing of TCR $\alpha$ or TCR $\beta$ transgenes with endogenous TCR $\beta$ or TCR $\alpha$ chains, respectively. TCR mis-pairing leads to the formation of unknown TCR specificities, which dilute the surfaceexpression of the therapeutic TCR $\alpha \beta$ heterodimer and can potentially result in off-target toxicity. Although, there is no clinical evidence for TCR mis-pairing-induced autoreactivity, preclinical data derived from a mouse model demonstrate that TCR mis-pairing can lead to graft-versus-host disease [10]. Strategies that prevent TCR mis-pairing are therefore expected to improve $\mathrm{T}$ cell avidity by increasing the level of cell surface expression of therapeutic TCR $\alpha \beta$ heterodimer and at the same time reduce potential off-target toxicity, reviewed in Govers et al. [11]. These strategies include the murinization of TCR $\alpha$ and $-\beta$ constant domains [12], introduction of additional cysteine residues in $\operatorname{TCR} \alpha$ and $-\beta$ to form an extra disulfide bridge [13], the exchange of structurally important amino acids between $\operatorname{TCR} \alpha$ and $-\beta$ [14], or the replacement of parts of TCR $\alpha$ and $-\beta$ constant domains

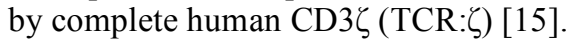

In previous studies, TCR: $\zeta$ has been extensively characterized regarding its ability to address TCR mis-pairing and 
functionally retarget $\mathrm{T}$ cells toward several tumor and virus antigens, such as MAGE-1/HLA-A1, gp100/HLA-A2, BMFL-1/HLA-A2, JC/HLA-A2 and EBNA-4/HLA-A11 [15-20]. Notably, TCR:ל's surface expression, which is enhanced when compared to wt TCR, and its ability to form immune synapses are independent of endogenous CD3 proteins. This receptor, possibly as a consequence of conformational changes, results in enlarged synapse sizes in TCRtransduced T cells $[18,20]$. Despite these unique properties, TCR: $\zeta$ and wt TCR do not differ with respect to the molecular 'make-up' of immune synapses and their ability to mediate antigen-specific $\mathrm{T}$ cell functions.

In the current study, we set out to minimize the content of $\mathrm{CD} 3 \zeta$ present in TCR: $\zeta$, specific for MAGE-A1/HLA-A1, without compromising the pairing and functional properties of TCR: $\zeta$, and with the intent to potentially decrease the immunogenicity of this receptor. In analogy to a study by Sommermeyer and colleagues, who defined a limited number of amino acids that preserved the benefits of murinized TCRs [21], the present effort would define those domains or amino acids of $\mathrm{CD} 3 \zeta$ responsible for improved TCR pairing and function. To generate minimal TCR: $\zeta$ variants we applied a domain-exchange as well as a 3-dimensional (3D) modeling strategy, and tested variants for TCR pairing and functional expression. We observed that the CD3 $\zeta$ transmembrane domain, and not a limited number of structurally defined amino acids, is critical for TCR: $\zeta$ 's surface expression and its inability to mis-pair with endogenous TCR chains, whereas the intracellular $\mathrm{CD} 3 \zeta$ domain is critical for $\mathrm{T}$ cell activation. A minimal TCR: $\zeta$ variant that lacked the extracellular $\mathrm{CD} 3 \zeta$ domain was best at preserving both TCR pairing and function in Jurkat $\mathrm{T}$ cells, but was not functionally expressed in primary $\mathrm{T}$ cells.

\section{MATERIALS AND METHODS}

\section{Cells and Reagents}

Jurkat T cell clone 19 [18], which expresses MelA/HLAA2 (MelA) TCR, and EBV-transformed B cell blasts (APD) was cultured in RPMI 1640 medium (BioWhittaker, Verviers, Belgium) containing 10\% Fetal Bovine Serum (FBS, Stonehouse, Gloucestershire, UK), streptomycin (100 $\mu \mathrm{g} / \mathrm{ml})$ and penicillin $(100 \mathrm{U} / \mathrm{ml})$ (Jurkat $\mathrm{T}$ cell medium). The human embryonic kidney cell line 293T, the packaging cell line Phoenix-A, and melanoma cell line Mel2A were cultured in DMEM medium (BioWhittaker) supplemented with $10 \%$ FBS, $2 \mathrm{mM}$ L-glutamine, non-essential amino acids and antibiotics. T lymphocytes derived from healthy donors were isolated and expanded as described elsewhere [22] and cultured in HEPES buffered RPMI 1640 medium supplemented with 10\% Human Serum, 2 mM L-glutamine, streptomycin and penicillin. Monoclonal Abs included: FITC- and non-conjugated anti-TCR-V $19 \mathrm{mAb}$ (Pierce Biotechnology, Inc., Rockford, IL); PE- and non-conjugated anti-TCR V 327 mAb (BD Biosciences, San Jose, USA); PEand non-conjugated anti-TCR-V $\beta 9 \mathrm{mAb}$ (BD Biosciences and Coulter-Immunotech, Marseille, France, respectively); PE-conjugated anti-CD107a mAb (BD biosciences); APCand non-conjugated anti-CD3 $\varepsilon$ mAb (OKT3) (BD Biosciences and Coulter-Immunotech, respectively); Cy5- and non-conjugated Rabbit-anti-Mouse ( $\mathrm{R} \alpha \mathrm{M})$ IgG Fab (Jackson
ImmunoResearch, Suffolk, UK). Peptide/MHC (pMHC) monomers included: MAGE-1 (M1: EADPTGHSY)/HLAA*0101; and Melan-A (MelA: ELAGIGILTV)/HLAA*0201 biotinylated peptide/MHC monomers (Sanquin, Amsterdam, Netherlands), and these pMHC monomers were tetramerized as described previously [23]. Other reagents included: Retronectin (human fibronectin fragments CH-296, Takara Shuzo Co. Ltd., Otsu, Japan); PMA (Sigma-Aldrich, St. Louis, USA); PHA (Remel Europe, Dartford, England); golgistop (BD biosciences) and streptavidin-PE (BD biosciences).

\section{Cloning of Minimal TCR: $\zeta$ Variants}

We have generated a panel of 11 TCRs, as schematically represented in (Fig. 1), that are specific for MAGE-A1/HLAA1 (M1/A1) and have the TCR-V gene usage TRAV19/J39/C and TRBV9/D2/J2-3/C2 (with TCR-V(D)J gene nomenclature according to http://imgt.cines.fr), originally derived from CTL clone MZ2-82/30 as described previously [15]. Control TCRs $(n=3)$ included wt TCR [18], TCR: $\zeta[15,18]$ and a murinized plus cysteine-modified TCR (i.e., TCR:mu+cys), the latter designed according to Cohen and colleagues [24]. TCR:mu+cys was generated via overlap PCR to fuse together M1/A1 TCR-V and murine TCR-C domains $(\mathrm{V} \alpha-\mathrm{mC} \alpha$ and $\mathrm{V} \beta-\mathrm{mC} \beta 2)$, which were ligated in pBullet vectors [15] via SalI-XhoI (TCR $\alpha)$ and NcoI-XhoI (TCR $\beta)$. Cysteine mutations (TCR $\alpha$ T189, and TCR $\alpha$ S191) were generated using QuickChange Site-Directed Mutagenesis Kit (Fynnzymes, Espoo, Finland) according to the manufacturer's instructions. The minimal TCR: $\zeta$ variants $(n=8)$ were generated via either one of the two approaches. First, six variants were made using a domain-exchange strategy in which extracellular $(\mathrm{ec})$, transmembrane (tm) or intracellular (ic) domains of $\mathrm{CD} 3 \zeta$, and combinations of these domains, were exchanged for corresponding TCR domains. These six variants were named as follows: TCR: $\zeta \Delta \mathrm{ec} ; \mathrm{TCR}: \zeta \Delta \mathrm{tm}$;

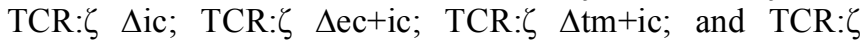
$\Delta \mathrm{ec}+\mathrm{tm}$ ( $\Delta$ indicates lack of $\mathrm{CD} 3 \zeta$ domain(s), and replacement by corresponding TCR domain(s)). The exact boundaries of ec, tm and ic domains of CD3 $\zeta$, TCR $\alpha$ and TCR $\beta$ are provided in the legend to (Fig. 1A). These variants were generally constructed by overlap PCR and gene synthesis, as described in detail in Supplementary Methods. Second, two additional TCR: $\zeta$ variants were made using a 3D-modeling strategy in which a limited number of amino acids of TCR tm were exchanged for $\mathrm{CD} 3 \zeta$ tm amino acids at structurally favorable positions. These two variants were named as follows: TCR $\operatorname{tm} \zeta 1$ and 2 , with the exact tm sequences provided by (Fig. 1B). The modeling software to design these variants, and their construction, generally by gene synthesis, is described in detail in Supplementary Methods. All TCR constructs made $(n=11)$ were sequence verified (Service XS, Leiden, Netherlands).

\section{Retroviral TCR Gene Transfer into T Cells}

TCR cDNAs were used to transduce Jurkat T cell clone 19 as well as human PBMC. To this end, Moloney Murine Leukemia retroviruses were produced by a co-culture of the packaging cells 293T and Phoenix-A following calciumphosphate transfections $[16,18]$. Packaging cells were trans- 
A

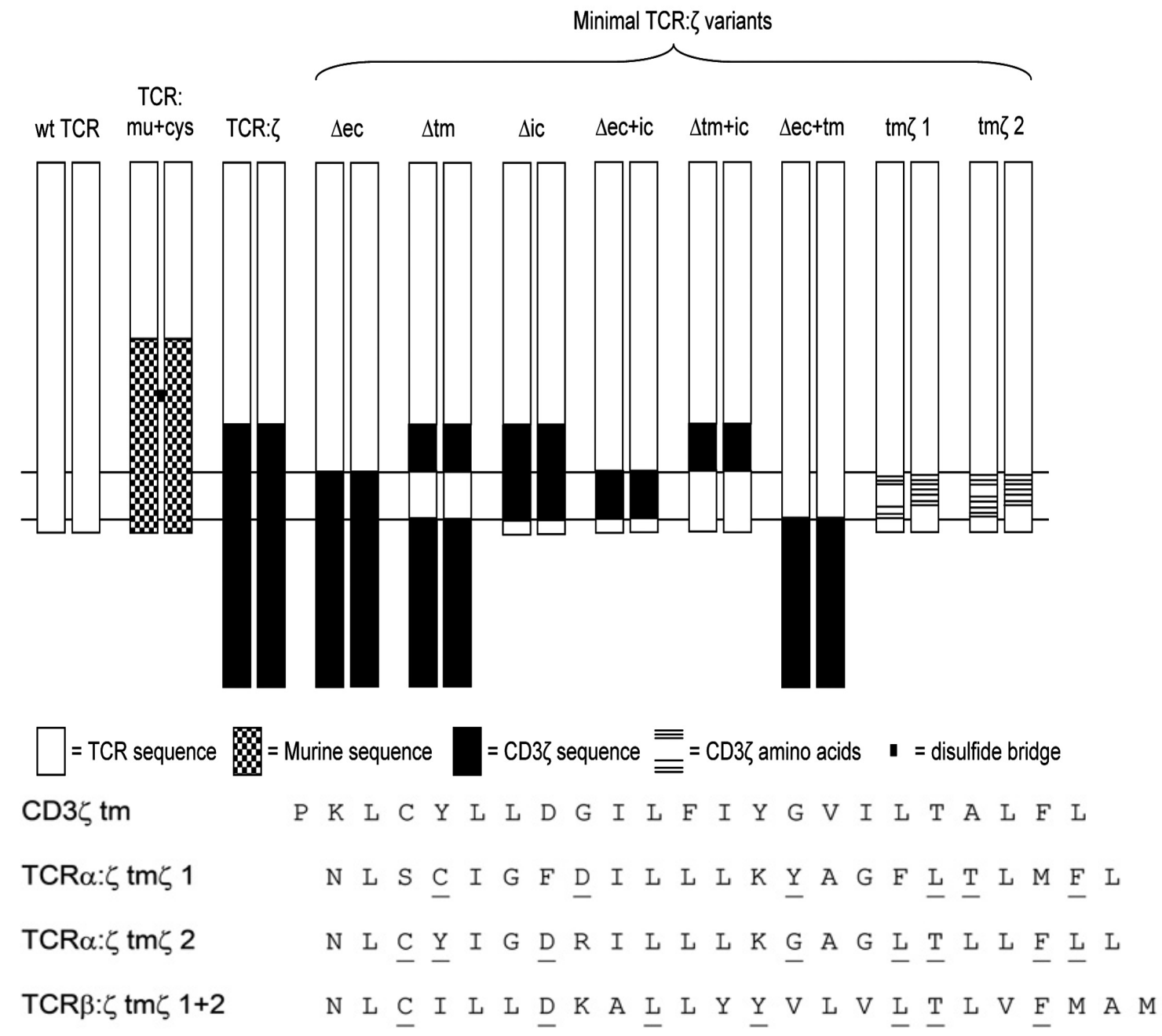

Fig. (1). Scheme of minimal TCR: $\zeta$ variants. (A) Minimal TCR: $\zeta$ constructs were modified via replacement of either extracellular $(\Delta \mathrm{ec})$, transmembrane $(\Delta \mathrm{tm})$, intracellular $(\Delta \mathrm{ic})$, extra and intracellular $(\Delta \mathrm{ec}+\mathrm{ic})$, transmembrane and intracellular $(\Delta \mathrm{tm}+\mathrm{ic})$ or extracellular and transmembrane $\mathrm{CD} 3 \zeta$ domains $(\Delta \mathrm{ec}+\mathrm{tm})$ (black) by corresponding TCR $\alpha$ and TCR $\beta$ domains (white). Boundaries for the CD3 $/ \mathrm{TCR} \alpha \beta$ ec, tm and ic domains were defined as follows. CD3 (genbank accession number: CAI21380.1) ec, tm and ic: nt 73-90 (aa 25-30); nt 91-153 (aa 31-51); and nt 154-489 (aa 52-163). TCR $\alpha$ [48] ec, tm and ic: nt 703-765 (aa 235-255); nt 766-825 (aa 256-275); and nt 826-840 (aa 276280). TCR $\beta$ [48] ec, tm and ic: nt 790-846 (aa 264-282); nt 847-912 (aa 283-304); and nt 913-933 (aa 305-311). In addition, two minimal TCR: $\zeta$ constructs were designed in which defined amino acids of tm CD3 $\zeta$ were transplanted onto structurally favourable positions in TCR $\alpha$ and TCR $\beta$ tm domains, and were termed minimal TCR:tm $\zeta 1$ and 2 (See Fig. (1B)). Control TCRs include: wt TCR, TCR: $\zeta$ and a TCR containing murine constant domains and additional cysteines (TCR:mu+cys, as described in [24]). (B) Transmembrane amino acids of CD3 $\zeta$ and minimal TCR: $\zeta$ variants TCR $\alpha: \operatorname{tm} \zeta 1, \mathrm{TCR} \alpha: \operatorname{tm} \zeta 2$, and TCR $\beta: \operatorname{tm} \zeta(\mathrm{CD} 3 \zeta$ amino acids underlined).

fected with TCR cDNAs, pHIT60 MLV GAG/POL, and VSV-G envelope plasmids for Jurkat $\mathrm{T}$ cell transductions, or with TCR cDNAs, pHIT60 MLV GAG/POL, and pCOLTGALV-envelope encoding plasmids for human PBMC transductions. The transduction procedure used was optimized for human $\mathrm{T}$ cells and described previously [25].

\section{Flow Cytometry and FACsort}

TCR-transduced $\mathrm{T}$ cells $\left(1 \times 10^{4}\right.$ cells $)$ were monitored by flow cytometry for surface expression of transgenic TCR using FITC-conjugated anti-TCR-V $\alpha 19$, PE-conjugated antiTCR-V $\beta 9$ mAbs and/or R-PE-conjugated M1/A1 tetramer; endogenous TCR (in case of Jurkat T cells) using PEconjugated anti-TCR-V $\beta 27 \mathrm{mAb}$ and/or R-PE-conjugated MelanA/A2 tetramer; and endogenous CD3 using APCconjugated anti-CD3 $\varepsilon \mathrm{mAb}$. After T cells were washed, they were incubated with $\mathrm{mAbs}$ for $30 \mathrm{~min}$ on ice, or $15 \mathrm{~min}$ at RT for pMHC tetramers. Next, T cells were washed and fixed with 1\% PFA (Brunschwig, Amsterdam, the Netherlands) before measurements on a FACSCalibur dual-laser flow cytometer (Beckton Dickinson, Alphen a/d Rijn, the Netherlands). Samples were analyzed using BD Cellquest software and displayed as dotplots or histograms. Enrichment of M1/A1 TCR-expressing Jurkat Clone $19 \mathrm{~T}$ cells was performed by two-color Fluorescent Activated Cell Sorting (FACS) following staining with FITC conjugated anti-TCRV $\alpha 19$ and PE conjugated anti-TCR-V $\beta 9$ mAbs.

\section{Flow Cytometry-Based FRET}

PE-labeled mAbs were used as donor and Cy5-labeled $\mathrm{R} \alpha \mathrm{M}$ antibody as acceptor and used in the following donor/acceptor combinations (as described in [18]): anti-TCR- 
$\mathrm{Vb} 27^{\mathrm{PE}}+$ anti-TCR-V $\alpha 19 / \mathrm{G} \alpha \mathrm{M}^{\mathrm{Cy} 5}$ and anti-TCR-Vb9 $9^{\mathrm{PE}}+$ anti-CD3 $\varepsilon(\mathrm{OKT} 3) / \mathrm{G} \mathrm{M}^{\mathrm{Cy} 5}$. Staining was performed sequentially, with extensive washing steps in between, using the following order of staining steps: first non-conjugated mAbs, second Cy5-labeled Abs, and last PE-labeled donor Abs. Fluorescence intensities of emissions at $570 \mathrm{~nm}$ (donor channel, excitation at $488 \mathrm{~nm}$ ), $670 \mathrm{~nm}$ (acceptor channel, excitation at 635), and over 670 (FRET channel, excitation at $488 \mathrm{~nm}$ ) were measured and collected on a FACSCalibur. Data were analyzed with the FLEX software on a per-cell basis [26].

\section{NFAT Reporter Gene Assay}

Gaussia-Luciferase reporter gene under the control of 6 NFAT response elements, a minimal IL-2 promotor, and a TATA box (in short: GLuc-NFAT(RE)6) was used to quantify TCR-mediated stimulation. Gluc-NFAT(RE)6 was generated by digesting a plasmid containing NFAT-6-luc [27] with NcoI-HinDIII and ligating this fragment (containing the 6 NFAT response elements, minimal IL-2 promotor and TATA box), together with a EcoRI-NcoI linker, in EcoRIHinDIII digested pGluc-basic vector (New England Biolabs, Ipswich, USA). $5 \times 10^{6}$ Jurkat TCR-transduced T cells were transiently nucleofected with an Amaxa nucleofector (AmaxaBiosystems, Cologne, Germany) according to previous optimizations [28,29]. Briefly, T cells were resuspended in $100 \mu \mathrm{l}$ supplemented buffer $\mathrm{V}$ to which $5 \mu \mathrm{g}$ GLucNFAT(RE)6 was added and pulsed with the Nucleofector set at program C-16. Next, T cells were immediately transferred to $2.5 \mathrm{ml}$ warm Jurkat T-cell medium in T25 flasks for $\mathrm{O} / \mathrm{N}$ recovery at $37^{\circ} \mathrm{C}$ and $5 \% \mathrm{CO}_{2}$. Non-tissue culture-treated 96 well plates were coated with non-conjugated $\mathrm{mIg} \mathrm{Ab}$, antiTCR-VB9 mAb (100 ng) or biotinylated M1/A1 or JC/A2 monomers (titrated from $126 \mathrm{nM}$ down to $4 \mathrm{nM}$ ). The latter added to streptavidin-coated plates $\mathrm{O} / \mathrm{N}$ at $4{ }^{\circ} \mathrm{C}$. Twenty hours post-transfection, $0.2 \times 10^{6} \mathrm{~T}$ cells at a concentration of $1 \times 10^{6} / \mathrm{ml}$ were transferred to each well in the 96 -well plates and stimulated for 6 hours at $37^{\circ} \mathrm{C}$ and $5 \% \mathrm{CO}_{2}$. Subsequently, $25 \mu \mathrm{l}$ supernatant was transferred to 96 wellplate read-out plates (Corning incorporated, Costar assay plate, Lowell, USA) and placed in a luminometer. Next, $50 \mu 1$ assay buffer was added (Gluc substrate; New England Biolabs, Ipswich, USA). Light units indicative of the Gaussia Luciferase-mediated enzymatic transition of coelenterazine into coelenteramide were measured according to the manufacturer's instructions (Mediators, Vienna, Austria) and expressed (in RLU) relative to wt TCR luciferase activities ( $100 \%$ for each concentration)

\section{CD107a Mobilization Assay}

TCR-transduced human PBMC $\left(1 \times 10^{6}\right)$ were resuspended in $0.15 \mathrm{ml}$ T cell medium, $0.5 \mu \mathrm{l}$ Golgistop (BD biosciences), and $100 \mu \mathrm{l}$ anti-CD107a-PE (BD biosciences). Mel2A and APD target cells $\left(1 \times 10^{6}\right)$ were resuspended in $0.25 \mathrm{ml} \mathrm{T}$ cell medium ( $\pm 10 \mu \mathrm{M}$ M1 peptide). Next, $50 \mu \mathrm{l}$ target cells and $50 \mu \mathrm{T}$ cells were mixed in a tissue culture treated 96-well plates (Greiner bio-one) and incubated for 2 $\mathrm{h}$ at $37^{\circ} \mathrm{C}$ and $5 \% \mathrm{CO}_{2}$. After the cells were washed, they were stained with anti-V $\alpha 19$-FITC and anti-CD3 $\varepsilon$-APC for 30 minutes at $4{ }^{\circ} \mathrm{C}$ in the dark. Next, cells were washed and fixed with $1 \%$ PFA and measured on a FACS Calibur dual- laser flow cytometer. T cells gated for viability (FSC and SSC) and positive for CD3 $\varepsilon$ and TCR-V $\alpha 19$, were assessed for surface expression of CD107a.

\section{Statistical Analyses}

Differences among TCRs in various assays were tested with student's t-tests (unpaired; two-tailed) using Graphpad Prism 4 software. Differences with $p$ values $<0.05$ were considered significant.

\section{RESULTS}

\section{CD3 $\zeta$ Transmembrane Domain is Required for Surface Expression of TCR: $\zeta$}

Jurkat $\mathrm{T}$ cells containing MelA/A2 TCR were used as recipient cells for a panel of $6 \mathrm{M} 1 / \mathrm{A} 1$ minimal TCR: $\zeta$ 's, each incorporating a different but minimal of ec, tm and/or ic CD3 $\zeta$ domains (see Fig. (1) for details). These dual-TCR Jurkat $\mathrm{T}$ cells were assessed for surface expression of transgenic TCR by flow-cytometry. Minimal TCR: $\zeta \Delta \mathrm{ec}, \Delta \mathrm{ic}$, $\Delta \mathrm{ec}+\mathrm{ic}$, all containing the $\mathrm{CD} 3 \zeta \mathrm{tm}$ domain, revealed a surface expression pattern like that of parental TCR: $\zeta$ with a typical diagonal, high mean fluorescence intensity (MFI) and absence of single TCR-chain positive cells (see Fig. (2A); [18]). TCR: $\zeta \Delta \mathrm{ic}$ and $\Delta \mathrm{ec}+\mathrm{ic}$ revealed lower levels of surface

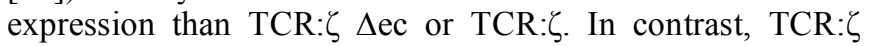
$\Delta \mathrm{tm}, \Delta \mathrm{tm}+\mathrm{ic}$ and $\Delta \mathrm{ec}+\mathrm{tm}$ transduced Jurkat $\mathrm{T}$ cells showed no surface expression of either or both TCR $\alpha: \zeta$ and TCR $\beta: \zeta$ chains (Fig. 2A). Further analysis of these latter TCRs revealed that mRNA, but not intracellular proteins were consistently present, suggesting that not gene transcription but more likely protein translation and/or transportation to the cell surface were hampered (Supplementary Fig. 1). Notably, the TCR:mu+cys, a murinized and cysteine-modified TCR used as a control TCR, shows an extended diagonal surface expression pattern and no or less single TCR-chain positive $\mathrm{T}$ cells than wt TCR, similar to TCR: $\zeta$ or its $\mathrm{CD} 3 \zeta$ tm-containing variants (Fig. 2A) and suggestive for high preferential TCR pairing.

\section{CD3 $\zeta$ Transmembrane Domain Critically Determines the Inability of TCR: $\zeta$ to Associate With Endogenous CD3 and TCR Chains}

The minimal TCR: $\zeta$ variants were assessed for their inability to associate with endogenous $\mathrm{CD} 3$, considered a unique characteristic of TCR: $\zeta$, using conventional flow cytometry and flow cytometric Fluorescence Resonance Energy Transfer (FRET). Flow cytometry analyses after double-staining for TCR $\beta$ and $\mathrm{CD} 3 \varepsilon$ revealed diagonal dotplots in Jurkat T cells expressing wt TCR and TCR:mu+cys, but not TCR: $\zeta$ or any of its minimal TCR variants (Fig. 3A). These data suggest on one hand a CD3-independence of minimal TCR: $\zeta$ variants that contain $\mathrm{CD} 3 \zeta \mathrm{tm}$, similar to the reported CD3-independence of TCR: $\zeta$, and confirm on the other hand a CD3-dependence of wt TCR and TCR:mu+cys [11]. The lack of competition for CD3-proteins by TCR: $\zeta$ $\Delta \mathrm{ec}, \Delta \mathrm{ic}, \Delta \mathrm{ec}+\mathrm{ic}$ was also reflected in the expression of endogenous TCR. TCR: $\zeta$ or these variants did not alter the MFI of endogenous TCR $\beta$, whereas wt TCR or TCR:mu+cys approximately halved the MFI of endogenous TCR $\beta$ (data not shown). Subsequent studies focused only on the three 
A

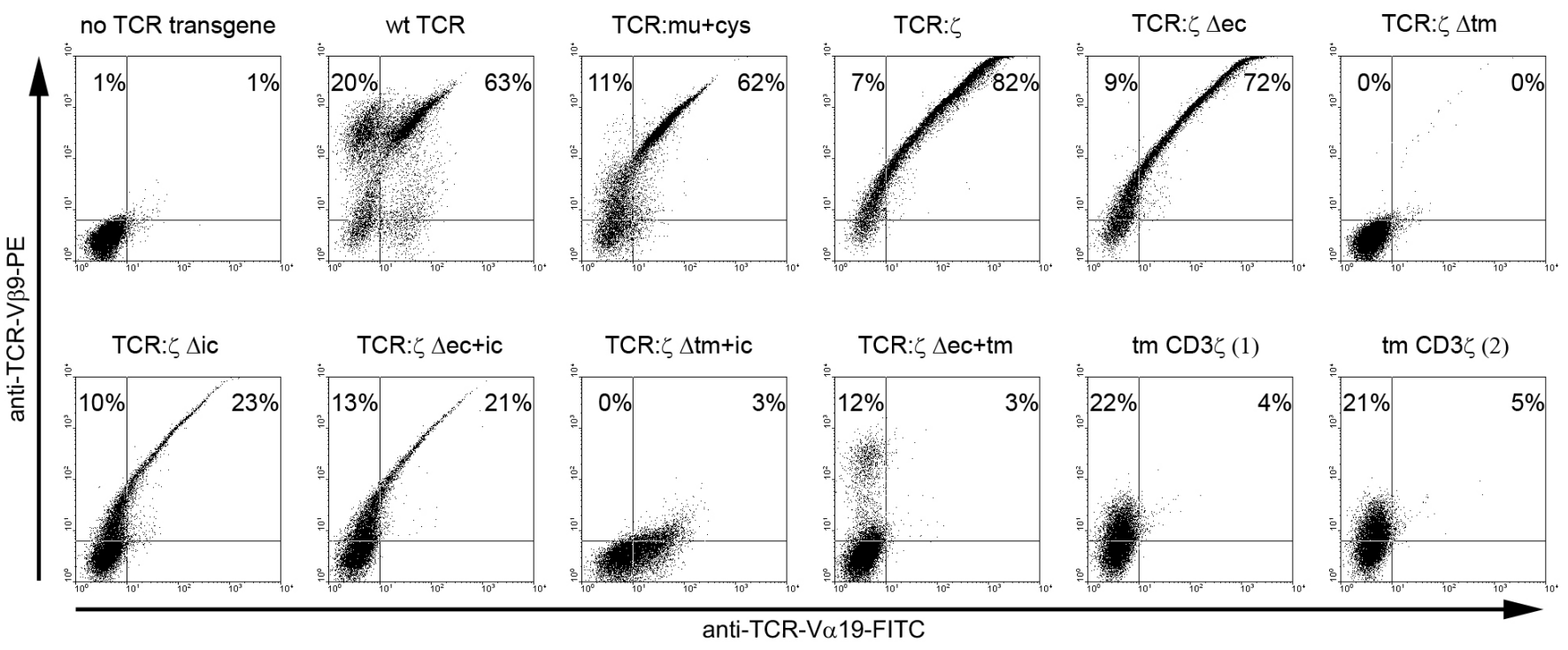

B

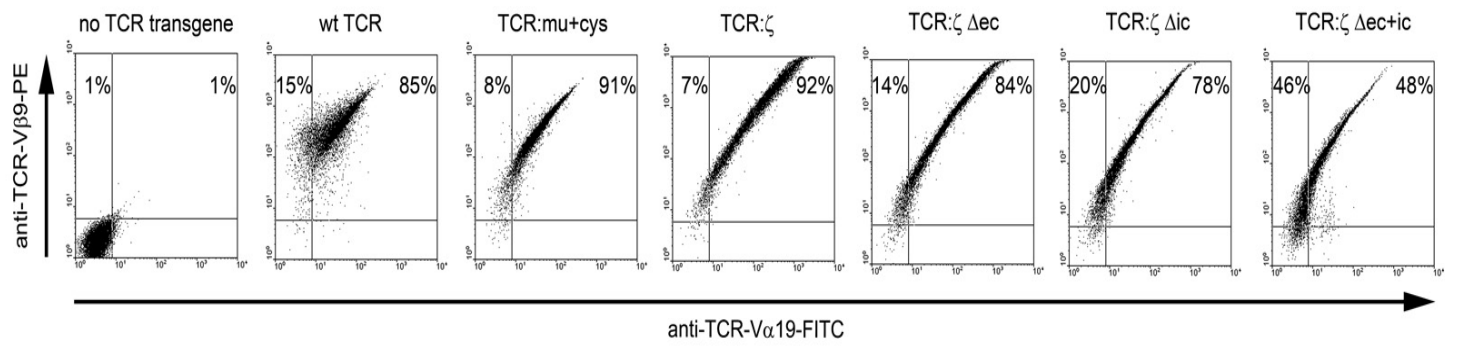

C

\begin{tabular}{l|c|c|c|c|c|c|} 
& wt TCR & TCR:mu+cys & TCR: $\zeta$ & TCR: $\zeta \Delta$ ec & TCR: $\zeta \Delta$ ic & TCR: $\zeta \Delta$ ec+ic \\
\hline MFI TCR-V $\alpha 19$ & $64 \pm 10$ & $66 \pm 11$ & $\begin{array}{c}242 \pm 46 \\
P<0.005\end{array}$ & $\begin{array}{c}163 \pm 37 \\
P<0.05\end{array}$ & $133 \pm 38$ & $76 \pm 26$ \\
\hline MFI TCR-V $\beta 9$ & $633 \pm 124$ & $540 \pm 108$ & $\begin{array}{c}1646 \pm 275 \\
P<0.005\end{array}$ & $1145 \pm 248$ & $998 \pm 265$ & $578 \pm 194$ \\
\hline $\begin{array}{l}\% \text { TCR } \alpha \beta \\
\text { heterodimer }\end{array}$ & $89 \pm 2.1$ & $92 \pm 1.1$ & $88 \pm 2.2$ & $\begin{array}{c}79 \pm 2.5 \\
P<0.05\end{array}$ & $\begin{array}{c}74 \pm 3 \\
P<0.005\end{array}$ & $\begin{array}{c}48 \pm 4.1 \\
P<0.0001\end{array}$ \\
\hline
\end{tabular}

Fig. (2). Minimal TCR: $\zeta \Delta \mathrm{ec}, \Delta \mathrm{ic}$ and $\Delta \mathrm{ec}+\mathbf{i c}$ express at T cell surface. Jurkat T cells expressing MelA/A2 TCR (Jurkat cl. 19) were transduced with one of the following M1/A1 TCRs: minimal TCR: $\zeta \Delta \mathrm{ec}, \Delta \mathrm{tm}, \Delta \mathrm{ic}, \Delta \mathrm{ec}+\mathrm{ic}, \Delta \mathrm{tm}+\mathrm{ic}, \Delta \mathrm{ec}+\mathrm{tm}, \mathrm{TCR} \operatorname{tm} \zeta 1, \mathrm{tm} \zeta 2, \mathrm{TCR}: \zeta$, wt TCR, TCR:mu+cys, or no TCR transgene. (A) Surface expression levels of transgenic MA1/A1 TCRs were measured via flow cytometry using anti-TCR-V $\alpha 19^{\mathrm{FITC}}$ and anti-TCR-V $\beta 9^{\mathrm{PE}} \mathrm{mAbs}$. Representative dotplots out of 5 individual measurements are displayed. See Supplementary Fig. 1. for extended analysis of intracellular protein and mRNA expression of those minimal TCR: $\zeta$ variants that did not show surface expression of both TCR-V $\alpha 19$ and TCR-V $\beta 9$, i.e., minimal TCR: $\zeta \Delta \mathrm{tm}, \Delta \mathrm{ec}+\mathrm{tm}, \Delta \mathrm{tm}+\mathrm{ic}, \mathrm{TCR}: \mathrm{tm} \zeta 1$ and tm $\zeta 2$. (B) TCR surface expression of Jurkat $\mathrm{T}$ cell lines transduced with minimal TCR: $\zeta \Delta \mathrm{ec}, \Delta \mathrm{ic}$ and $\Delta \mathrm{ec}+\mathrm{ic}$ or controls wt TCR, TCR: $\zeta$ and TCR:mu+cys after FACSort with anti-TCR-V $\alpha 19^{\mathrm{FITC}}$ and anti-TCR-V $\beta 9^{\mathrm{PE}} \mathrm{mAbs}$. Representative dotplots out of 5 individual measurements are displayed and percentages of stained T cells in upper left and upper right quadrants are indicated. (C) Mean Fluorescence Intensities or percentages (both + SEM) of TCR $\alpha \beta$ in upper right quadrants in (B), $n=6-11$ independent measurements (statistically significant differences in comparison to wt TCR are calculated with student's t-tests; p-values indicated in Fig).

minimal TCR: $\zeta$ variants that were expressed on the cell surface. Flow cytometric FRET confirmed lack of association between TCR: $\zeta$ or its minimal variants, but not wt TCR or TCR:mu+cys, and CD3e (Fig. 3B). Flow cytometric FRET was also applied to address the extent TCRs were prone to
TCR mis-pairing. Using antibodies specific for endogenous TCR-Vß27 (PE-fluorochrome, donor) and the TCR-V $\alpha 19$ transgene (Cy5-fluorochrome, acceptor), we observed no FRET signals above background for TCR: $\zeta \Delta \mathrm{ec}, \Delta \mathrm{ic}$, or $\Delta \mathrm{ec}+\mathrm{ic}$ variants, similar to parental TCR: $\zeta$, indicating that 
$\mathbf{A}$

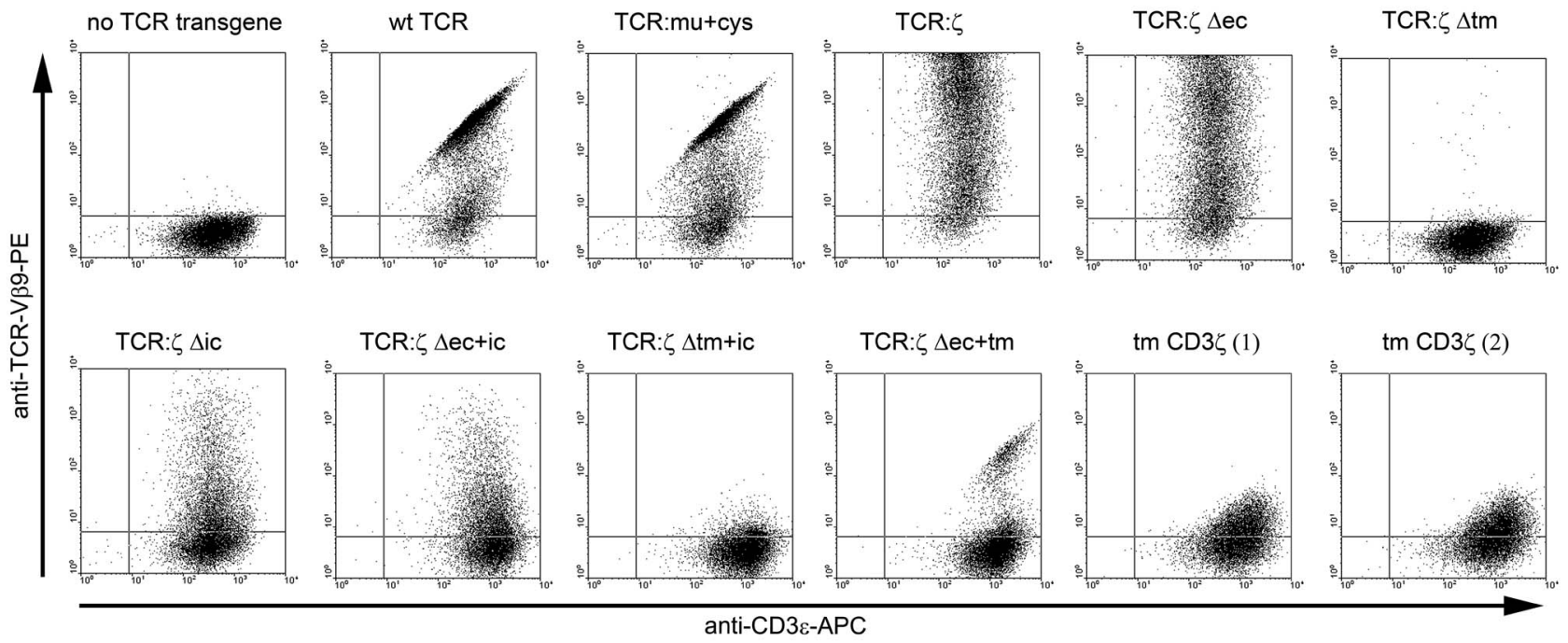

B

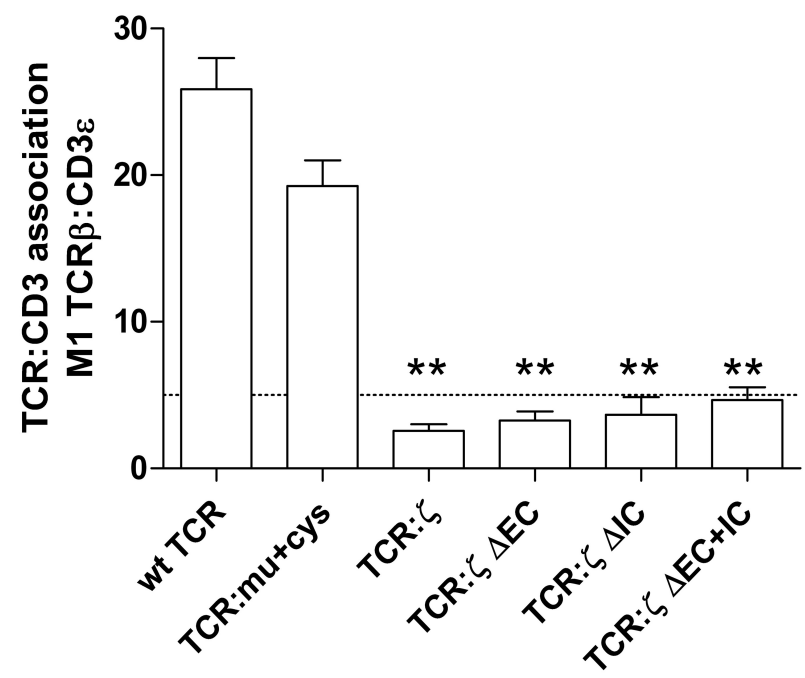

C

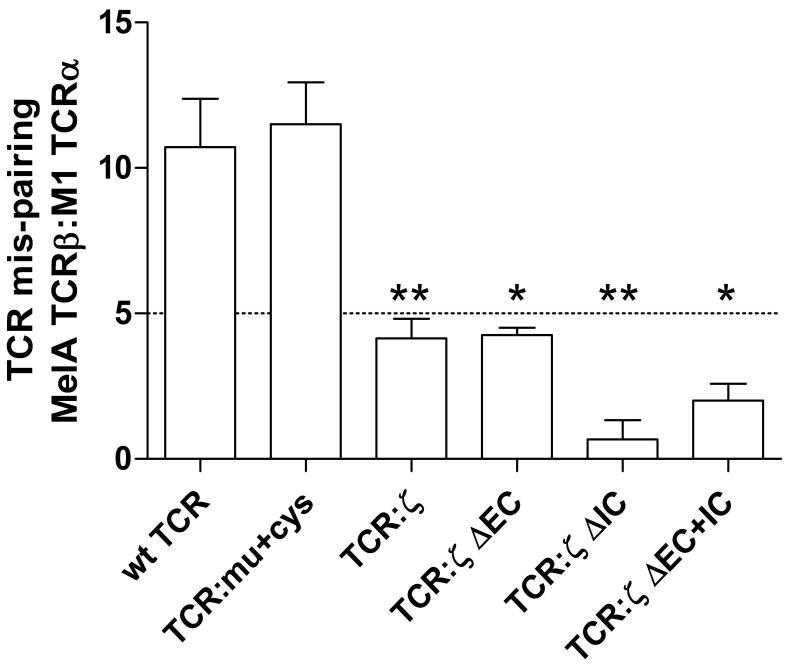

Fig. (3). Minimal TCR: $\zeta \Delta \mathrm{ec}, \Delta \mathrm{ic}$ and $\Delta \mathrm{ec}+\mathrm{ic}$ show neither CD3 association nor TCR mis-pairing. (A) Jurkat $\mathrm{T}$ cells expressing minimal TCR: $\zeta \Delta \mathrm{ec}, \Delta \mathrm{ic}$ and $\Delta \mathrm{ec}+\mathrm{ic}, \mathrm{TCR}: \zeta$, wt TCR, TCR:mu+cys transgenes were tested for surface expression of transgenic TCR and CD3 $\varepsilon$ via flow cytometry using anti-TCR-V $\beta 1^{\mathrm{PE}}$ and anti-CD3 $\varepsilon^{\mathrm{APC}}$ antibodies. Representative examples out of 5 individual measurements are displayed. (B) CD3 $\varepsilon$ association and (C) TCR mis-pairing were determined of minimal TCR: $\zeta \Delta \mathrm{ec}, \Delta \mathrm{ic}$ and $\Delta \mathrm{ec}+\mathrm{ic}$ and control TCRs using Fluorescence Resonance Energy Transfer (FRET) between anti-V $\beta 9^{\mathrm{PE}}->$ anti-CD3 $\varepsilon-\mathrm{R} \alpha \mathrm{M}^{\mathrm{Cy} 5}$ and anti-V $\beta 27^{\mathrm{PE}}->$ anti-V $\alpha 19-\mathrm{R} \alpha \mathrm{M}^{\mathrm{Cy} 5} \mathrm{mAbs}$, respectively. Please note that FRET was measured using TCR-transduced but non-sorted Jurkat T cells after gating on M1/A1-specific TCR expressing cells. Dotted line represents the level of background signal (5\%) and bars represent mean FRET values + SEM, $n=4$ independent measurements (statistically significant differences in comparison to wt TCR are calculated with student's t-tests; $*=\mathrm{p}<0.05 ; * *=\mathrm{p}<0.005$ ).

these variants preserve TCR: '’s ability to successfully address TCR mis-pairing (Fig. 3C). Notably, when using a sensitive methodology such as FRET, TCR:mu+cys TCR $\alpha$ are mis-paired with endogenous TCR $\beta$ to the same extent as wt TCR $\alpha$. Additional proof for the absence or presence of TCR mis-pairing came from single TCR chain transductions of Jurkat T cells. Supplementary Fig. 2 demonstrates significant cell surface expression of a TCR $\alpha \beta$ heterodimer (indicative of TCR mis-pairing) upon transduction with single chains (either TCR $\alpha$ or $-\beta$ ) of wt TCR or TCR:mu+cys, but not

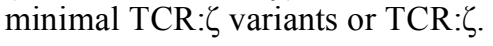

Next, we attempted to identify a limited number of CD3 $\zeta$ tm amino acids important for TCR: $\zeta$ 's surface expression and inability to mis-pair. A 3D-modeling strategy was applied to define a set of $\mathrm{CD} 3 \zeta$ transmembranal amino acids that was subsequently transplanted onto favorable positions in the transmembrane domain of TCR chains. This resulted in TCR: $\zeta$ variants $\operatorname{tm} \zeta 1$ and $-\operatorname{tm} \zeta 2$ which were retrovirally introduced in Jurkat $\mathrm{T}$ cells. Flow cytometry revealed no cell surface expression of minimal TCR:tm $\zeta 1$ or -2 heterodimers on these dual-TCR Jurkat $\mathrm{T}$ cells (Fig. 2A). TCR $\alpha: \operatorname{tm} \zeta 1$ and -2 chains were not able to complex with TCR $\beta: \operatorname{tm} \zeta$ or 
endogenous TCR $\beta$. Also, TCR $\beta: \operatorname{tm} \zeta$ showed moderate levels of surface expression through mis-pairing with the endogenous $\mathrm{TCR} \alpha$. Analysis of mRNA and intracellular protein suggested difficulties in protein transportation to the cell surface of particularly both TCR $\alpha$-chains (Supplementary Fig. 1). Thus, we were unable to attribute strong CD3-independent cell surface expression and prevention of TCR mis-pairing to a subset of $\mathrm{CD} 3 \zeta$ transmembranal amino acids.

\section{Minimal TCR: $\zeta \Delta$ ec Performs best at Preserving the Ability of TCR: $\zeta$ to Bind pMHC and Activate NFAT}

To study TCR-mediated functions, we have FACSorted Jurkat TCR-transductants with TCR $\alpha$ and $-\beta$ mAbs to enhance and equalize surface expression levels of the different TCR formats (Fig. 2B). Enrichment resulted in similar surface expression levels of TCRs (range between $74-92 \%$ ), except for TCR: $\zeta \Delta \mathrm{ec}+$ ic which fell behind (48\%) (Fig. 2C). MFIs were within the same range for TCR: $\zeta \Delta \mathrm{ec}, \Delta \mathrm{ic}$ and

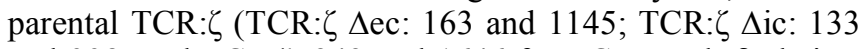

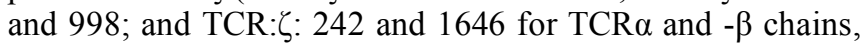
respectively), and were lowered for TCR: $\zeta \Delta \mathrm{ec}+\mathrm{ic}$ (76 and 578) (Fig. 2C). In fact, MFIs of TCR: $\zeta \Delta \mathrm{ec}+\mathrm{ic}$ were within the same range as those for wt TCR and TCR:mu+cys (wt TCR: 64 and 633; TCR:mu+cys: 66 and 540). Please note that levels of surface expression of TCR: $\zeta$ and its variants do not take into account TCR stainings in upper left quadrants of flow cytometry dot plots. Since these stainings extend the diagonal of flow cytometry dotplots and are not due to TCR mis-pairing (Fig. 3C), levels of surface expression, as put in Fig. 2C, may underestimate total surface expression levels of TCR: $\zeta$ and its variants.

Subsequently, transgenic TCRs were standardized for average cell surface expression levels (with \% surface expression of wt TCR (see (Fig. 2C)) set to 1.0) and compared for their ability to recognize and bind pMHC. The TCR: $\zeta$ $\Delta \mathrm{ec}$ transgene revealed a slightly lower percentage of pMHC binding compared to the TCR: $\zeta$ transgene, detected at all measured concentrations, although differences reached no statistical significance (Fig. 4A). Wild-type TCR, TCR:mu+cys, and TCR: $\zeta$ transduced Jurkat $T$ cells showed the highest percentages of pMHC positive populations (Fig. 4A). Again, when analyzing the MFIs of pMHC binding, we noted that Jurkat T cells expressing TCR: $\zeta \Delta \mathrm{ec}$ or parental TCR: $\zeta$ were the two $\mathrm{T}$ cell lines with the highest TCR expression levels (Figs. 4B and 2C). Although TCR: $\zeta \Delta \mathrm{ec}$ shows a pMHC

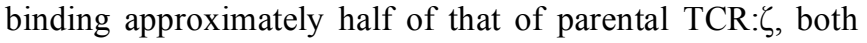
show a significantly increased MFI compared to wt TCR (TCR: $\zeta$ vs wt TCR: $p<0.05$ at all concentrations; and TCR: $\zeta$ $\Delta$ ec vs wt TCR: $p<0.05$ at 4 out of 6 concentrations). TCR: $\zeta$ $\Delta \mathrm{ic}$ and $\Delta \mathrm{ec}+\mathrm{ic}$ variants showed a negligible pMHC binding, whereas TCR:mu+cys showed a pMHC binding that was slightly lower than wt TCR.

Next, we measured antigen-specific $\mathrm{T}$ cell activation by employing a Gaussia Luciferase reporter assay based on six response elements of Nuclear Factor of Activated $\mathrm{T}$ cells (NFAT), a transcription factor that is a key step in $\mathrm{T}$ cell activation. With transgenic TCRs being standardized for average cell surface expression levels (Fig. 2C), we observed that TCR: $\zeta \Delta \mathrm{ec}$ and parental TCR: $\zeta$ proved to be most potent in activating Jurkat $T$ cells by inducing the highest level of luminescence, which was significantly higher when compared to wt TCR (Fig. 5). TCR: $\zeta \Delta \mathrm{ic}$ and $\Delta \mathrm{ec}+\mathrm{ic}$ variants, however, did not mediate $\mathrm{T}$ cell activation, even though these TCRs were able to bind pMHC. To further investigate the potency of TCR: $\zeta \Delta \mathrm{ic}$ and $\Delta \mathrm{ec}+\mathrm{ic}$ to induce intracellular $\mathrm{T}$ cell signaling, we stimulated Jurkat $\mathrm{T}$ cells with anti-TCRV $\beta 9$ mAbs and demonstrated that these two TCRs, both lacking the intracellular $\mathrm{CD} 3 \zeta$ immunoreceptor tyrosine-
A

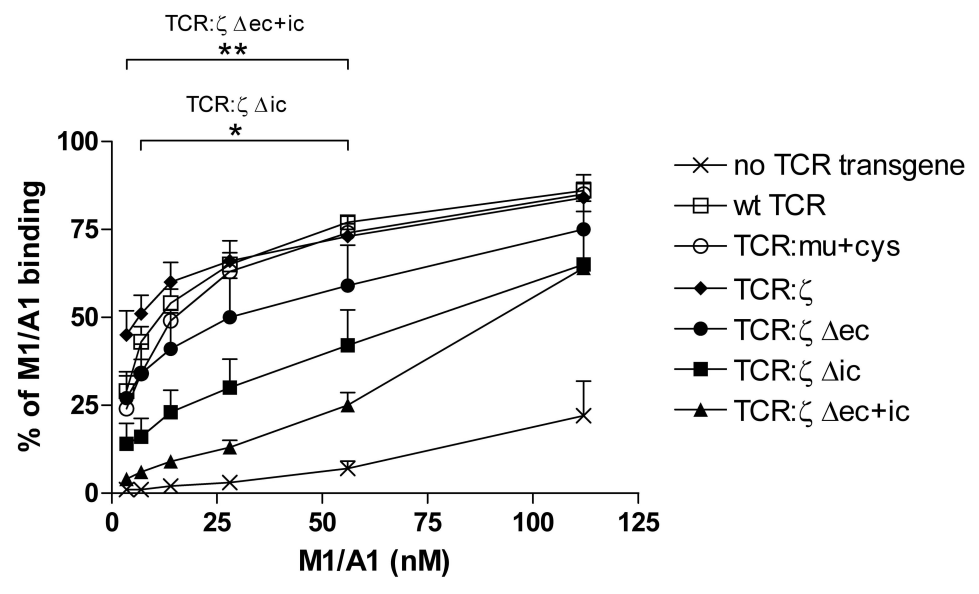

B

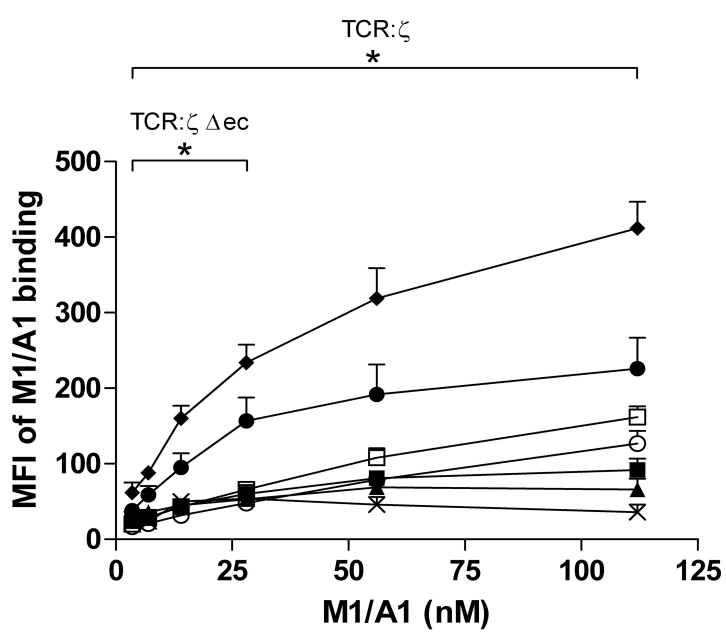

Fig. (4). T cells transduced with minimal TCR: $\zeta \Delta$ ec, $\Delta$ ic or $\Delta$ ec+ic show decreased ability to bind pMHC, which is least compromised for TCR: $\zeta \Delta$ ec. Jurkat T cells expressing minimal TCR: $\zeta \Delta$ ec, $\Delta$ ic and $\Delta$ ec $+\mathrm{ic}, \mathrm{TCR}: \zeta$, wt TCR, TCR:mu+cys transgenes or no TCR transgene were tested for their ability to bind M1/A1 tetramer PE complexes. The amount of M1/A1-tetramer used to stain $0.5 \mathrm{x} 10^{6}$ cells $(30$ min, RT) was titrated from $112 \mathrm{nM}$ down to $3.5 \mathrm{nM}$. Percentages (A) and MFIs (B) of pMHC binding by T cells were measured via flow cytometry. Percentages of pMHC binding were standardized for average cell surface expression levels (with \% surface expression of wt TCR (see Fig. (2C)), set to 1.0). Curves represent mean percentages of MFI or pMHC binding + SEM, $n=4$ independent measurements (statistically significant differences in comparison to wt TCR are calculated with student's t-tests; $*=p<0.05 ; * *=p<0.005$ ). 


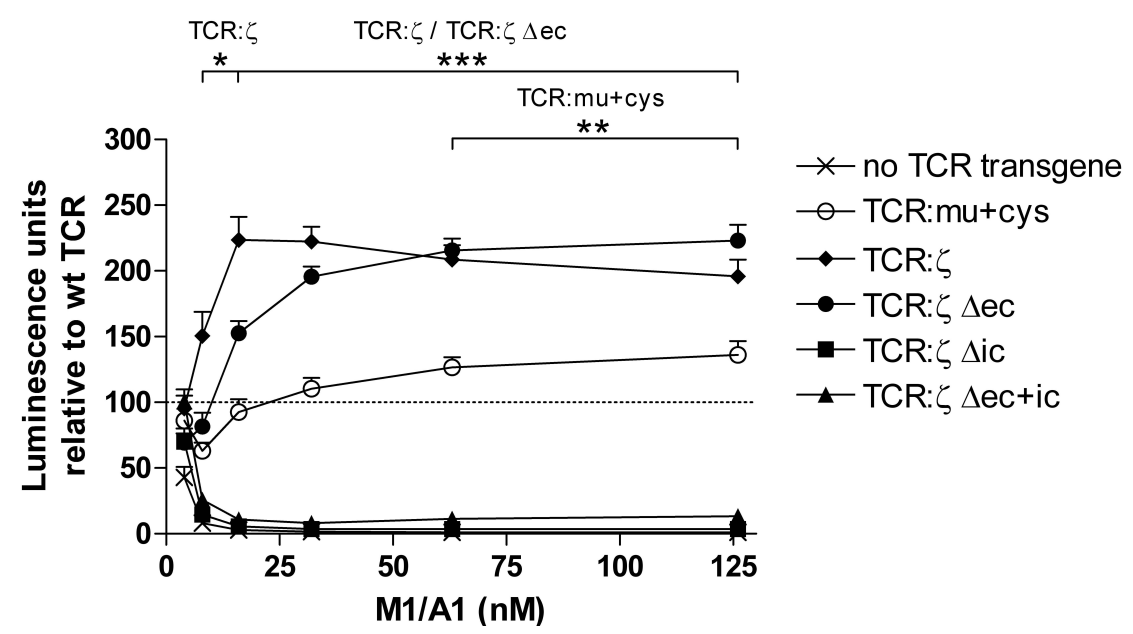

Fig. (5). $T$ cells transduced with minimal TCR: $\zeta \Delta$ ec show a potent antigen-specific NFAT response. Jurkat $T$ cells expressing minimal TCR: $\zeta \Delta \mathrm{ec}, \Delta \mathrm{ic}$ and $\Delta \mathrm{ec}+\mathrm{ic}, \mathrm{TCR}: \zeta$, wt TCR, TCR:mu+cys transgenes or no TCR transgene were tested for their ability to mediate activation of Nuclear Factor of Activated T cells (NFAT). TCR-transduced Jurkat T cells were nucleofected with a Gaussia Luciferase reporter construct under control of 6 NFAT response elements, and stimulated for $6 \mathrm{~h}$ with pMHC monomers. The concentration of monomers was titrated from $126 \mathrm{nM}$ down to $4 \mathrm{nM}$. Luciferase activities of wt TCR T cells for 4, 8, 16, 32, 63, and $126 \mathrm{nM}$ pMHC were: 588926, 670147, 656821, 444174, 181790, and 37084 relative luminescence units (RLU), respectively, and were all set at 100\% (dotted line). Luciferase activities of TCR-transduced T cells were standardized for average cell surface expression levels (as explained in legend to Fig. 4A). Curves represent mean luminescence units + SEM, $n=6-14$ independent measurements (statistically significant differences in comparison to wt TCR are calculated with student's t-tests; $*=\mathrm{p}<0.05, * * *=\mathrm{p}<0.0005)$.

based activation motifs (ITAMs), were not able to mediate T cell activation (Supplementary Fig. 3). Stimulations of the endogenous TCR $\beta$ with anti-TCR-V $\beta 27 \mathrm{mAb}$ did not show reduced NFAT activation upon transduction with TCR: $\zeta \Delta \mathrm{ec}$, $\Delta \mathrm{ic}$ or $\Delta \mathrm{ec}+\mathrm{ic}$, in line with parental TCR: $\zeta$ [18] (data not shown). In contrast, stimulation with anti-TCR-V $\beta 27 \mathrm{mAb}$ did show significantly reduced NFAT activation upon transduction with wt TCR or TCR:mu+cys, a result of downregulated surface expression of CD3-dependent TCRs in dual TCR T cells.

\section{Minimal TCR: $\zeta \Delta$ ec Shows a Compromised Surface Expression and Function in Primary Human T Cells}

The next step was to test the minimal TCR: $\zeta$ variant that performed best in Jurkat $\mathrm{T}$ cells with respect to surface expression, absence of TCR mis-pairing, pMHC binding and activation of NFAT, i.e., TCR: $\zeta \Delta \mathrm{ec}$, in primary human $\mathrm{T}$ cells. Anti-CD3 mAb-activated human PBMC were retrovirally transduced with TCR: $\zeta \Delta \mathrm{ec}, \mathrm{TCR}: \zeta$, wt TCR and TCR:mu+cys. To enhance the functional expression of TCR: $\zeta \Delta$ ec, we codon optimized $\operatorname{CD} 3 \zeta \Delta \mathrm{ec}$ and aligned the two chains in a TCR $\beta-2 \mathrm{~A}-\mathrm{TCR} \alpha$ configuration in a pMP71 retroviral vector [30-33]. Surface expression of TCR transgenes and pMHC binding were assessed by flow-cytometry in cultures of TCR-engineered T cells ( $>90 \%$ CD8-positive $\mathrm{T}$ cells). Please note that, for reasons explained above, percentages of surface expression displayed in the upper right quadrants of flow cytometry dotplots may provide an underestimation of the actual levels of surface expression of TCR: $\zeta$ and TCR: $\zeta \Delta \mathrm{ec}$ (with and without codon optimization) (Fig. 6A). TCR: $\zeta \Delta$ ec revealed a weak surface expression and pMHC binding, which was, unexpectedly, not improved when using TCR:opt. $\zeta \Delta \mathrm{ec}$ in a $\beta-2 \mathrm{~A}-\alpha$ configuration in pMP71 (Figs. 6A,B). Notably, the surface expression levels of TCR:५ and TCR:mu+cys were higher when com- pared to wt TCR (Fig. 6A). However, when looking at the pMHC binding, only TCR: $\zeta$, and not TCR:mu+cys, demonstrated enhanced performance when compared to wt TCR (Fig. 6B).

Finally, primary human T cells expressing TCR transgenes were stimulated with antigen-positive and negative target cells, after which CD107a mobilization on the cell surface was measured within the TCR-V 19 positive T cell population as a measure for cytotoxicity. TCR: $\zeta \Delta \mathrm{ec}$, whether or not with codon optimized CD3 $\zeta$ and in an optimal vector cassette, mediated negligible antigen-specific responses in contrast to TCR: $\zeta(p<0.005$, Fig. 6C). Both TCR: $\zeta$ and TCR:mu+cys show a higher percentage of CD107a positive cells than T cells with wt TCR transgenes.

\section{DISCUSSION}

In this study, we generated novel MAGE-A1-specific TCRs fused to partial rather than complete human CD3 $\zeta$ in an effort to identify a minimal TCR: $\zeta$ that preserved the TCR

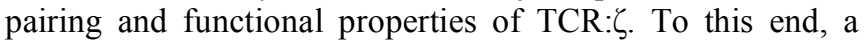
panel of 8 minimal TCR: $\zeta$ 's (see Fig. (1) for details) was tested for surface expression, association with endogenous CD3 and TCR chains, pMHC binding and TCR transgenemediated functions in Jurkat $\mathrm{T}$ cells and primary human $\mathrm{T}$ cells. Our observations revealed that for TCR: $\zeta$ and its variants: (a) intact $\mathrm{CD} 3 \zeta$ transmembrane (tm) domain critically determines surface expression and inability to associate with endogenous CD3 and TCR chains in Jurkat T cells; (b) both $\mathrm{CD} 3 \zeta$ extracellular (ec) and intracellular (ic) domains are dispensable for pMHC binding, whereas only CD3 $\zeta$ ec domain is dispensable for $\mathrm{T}$ cell mediated signaling in Jurkat $\mathrm{T}$ cells; and (c) the combination of CD3 $\zeta$ ec, tm and ic domains is required for surface expression and $\mathrm{T}$ cell function in primary human T cells. 
A

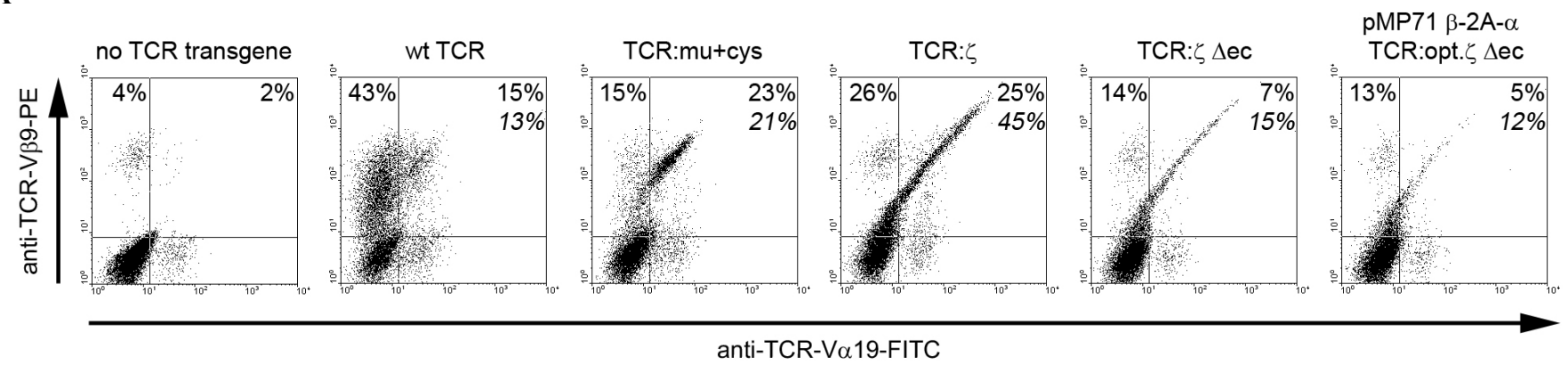

B

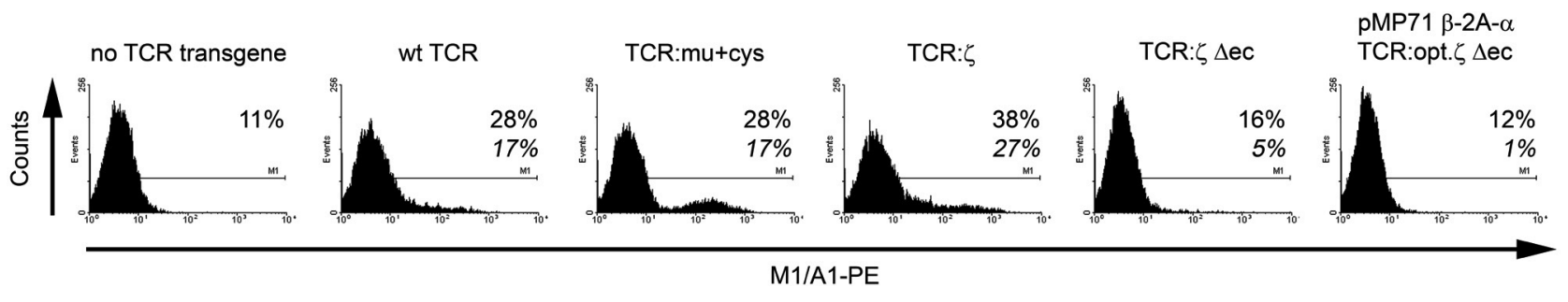

C

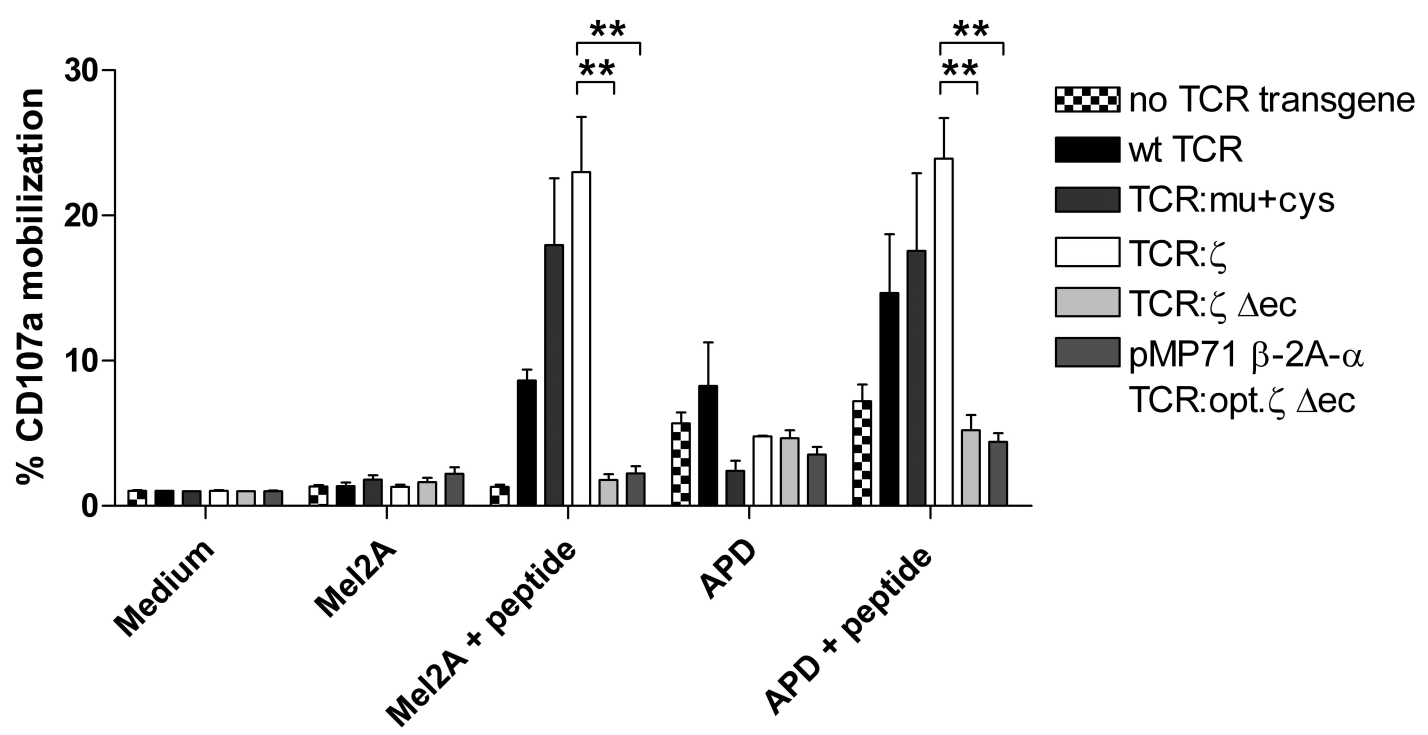

Fig. (6). Minimal TCR: $\zeta \Delta$ ec demonstrates compromised surface expression and antigen-specific function in primary human $T$ cells. Primary human T cells were transduced with one of the following M1/A1 TCRs: TCR: $\zeta \Delta$ ec, pMP71 $\beta-2 \mathrm{~A}-\alpha$ TCR:opt. $\zeta \Delta$ ec, TCR: $\zeta$, wt TCR, TCR:mu+cys, or no TCR. (A) Surface expression of transgenic TCR was measured via flow cytometry. Anti-TCR-V $\alpha 19^{\text {FITC }}$ and antiTCR-V $\beta 9^{\mathrm{PE}}$ antibodies were used to stain TCR-transduced T cells. Representative examples out of 5 individual measurements of two healthy donors are displayed and percentages of stained $\mathrm{T}$ cells in upper left and upper right quadrants are indicated. Percentages indicated in italics are either percentages of upper right quadrants (wt TCR, TCR:mu+cys) or the sum of percentages of upper left and upper right quadrants (TCR: $\zeta$, TCR: $\zeta \Delta \mathrm{ec}$, TCR:opt. $\zeta \Delta \mathrm{ec}$ ), corrected for the corresponding percentage(s) of Mock T cells. These percentages (in italics) may better represent the actual levels of surface expression of TCR: $\zeta$ and its variants (see Result section for details). (B) pMHC binding by transgenic TCR was measured via flow cytometry. M1/A1-tetramer-PE complexes (15 nM) were used to stain TCR-transduced T cells, and percentages of positive cells in histograms are indicated. Representative examples out of 5 individual measurements of two healthy donors are displayed and percentages of stained $\mathrm{T}$ cells in selected histogram region are indicated (in italics: corrected for pMHC binding observed by Mock T cells). (C) CD107a-mobilization to cell surface of TCR-transduced T cells. TCR-transduced T cells were stimulation for $2 \mathrm{~h}$ with medium, Mel2A or APD (both M1 negative, HLA-A1 positive), or Mel2A and APD loaded with $10 \mu \mathrm{M}$ M1 peptide, after which T cells were analyzed for CD107a expression by flow cytometry (see Materials and Methods section for details). Bars represent mean CD107a values + SEM, 2 independent measurements of two healthy donors (statistically significant differences are calculated with student's t-tests; $* *=\mathrm{p}<0.005$ ). Please note that at the time of flow cytometry analyses, TCR-engineered primary human T cells contained $>90 \%$ CD8-positive T cells. 
In the first series of experiments, we have analyzed 6 minimal TCR: $\zeta$ variants, in which the ec, tm and/or ic domain(s) of $\mathrm{CD} 3 \zeta$ were omitted (and replaced by corresponding TCR domain(s)). Cell surface expression analysis revealed that not all minimal TCR: $\zeta$ variants resulted in detection of a M1/A1 TCR heterodimer. In case the CD3 $\zeta \mathrm{tm}$ domain was absent (i.e., TCR: $\zeta \Delta \mathrm{tm}, \Delta \mathrm{tm}+\mathrm{ic}, \Delta \mathrm{ec}+\mathrm{tm}$ ), $\mathrm{TCR} \alpha: \zeta$ and $-\beta: \zeta$ transgenes were not properly expressed as a TCR heterodimer (Fig. 2A). In fact, TCR chains of these minimal TCR: $\zeta$ variants showed aberrant protein expression or transport to the cell surface (Supplementary Figs. 1B, C). $\mathrm{TCR}: \zeta$ and its variants that were expressed (i.e., TCR: $\zeta \Delta \mathrm{ec}$, $\Delta \mathrm{ic}$, and $\Delta \mathrm{ec}+\mathrm{ic}$, , show similarly strong levels of cell surface expression (TCR: $\zeta \Delta \mathrm{ec}+\mathrm{ic}$ to a lesser extent; Fig. 2) and an inability to associate with CD3e (Fig. 3A, B). Functional assays, however, identified two groups of TCRs: (1) TCR: $\zeta$ and TCR: $\zeta \Delta \mathrm{ec}$; and (2) TCR: $\zeta \Delta \mathrm{ic}$ and TCR: $\zeta \Delta \mathrm{ec}+\mathrm{ic}$. The latter group of TCRs, in contrast to the first group, revealed lowered to negligible binding of pMHC (Figs. 4A, B) and an inability to induce NFAT activity upon stimulation with either pMHC (Fig. 5) or anti-TCR-V $\beta 9$ mAb (Supplementary Fig. 3). Our finding that TCR: $\zeta$ variants that lack the $\mathrm{CD} 3 \zeta$ ic domain showed compromised pMHC binding is unexpected. Studies with other antigen-specific receptors argue that lack of, or not having access to, ITAM-bearing $\mathrm{CD} 3 \zeta$ ic domain results in enhanced surface expression and pMHC binding. For example, inhibition of protein tyrosine kinases demonstrated that early TCR-mediated signaling normally provides a negative feedback loop that facilitates pMHC-induced TCR down-regulation and T cell apoptosis [34, 35]. Moreover, studies with Chimeric Antibody Receptors coupled to $\mathrm{CD} 3 \zeta$ (i.e., CAR: $\zeta$ ) showed that $\mathrm{CD} 3 \zeta$ ITAMs directly contribute to loss of transgene expression and enhanced sensitivity to apoptosis [36]. In contrast, $\mathrm{TCR}: \zeta$ is different from other receptors since it does not dimerize with endogenous TCR/CD3 complexes [18] and may signal differently [37]. Moreover, recent studies into immune synapses suggested that TCR: $\zeta$ has a distinct conformation [20], and we propose that conformational changes due to extensive deletions, such as $\Delta \mathrm{ic}$ and $\Delta \mathrm{ec}+\mathrm{ic}$, cause the observed decrease in pMHC binding. The observation that TCR: $\zeta \Delta \mathrm{ic}$ and $\Delta \mathrm{ec}+\mathrm{ic}$ can not mediate activation of NFAT, even upon stimulation with anti-TCR mAb, points out that TCR-mediated signaling either requires a modified TCR that contains ITAMs (i.e., TCR: $\zeta$ or minimal TCR: $\zeta \Delta \mathrm{ec}$ ) or a (modified or wt) TCR that is able to recruit endogenous and ITAM-containing CD3 molecules.

In addition to the shared inability to associate with $\mathrm{CD} 3 \varepsilon$, minimal TCR: $\zeta \Delta \mathrm{ec}, \Delta \mathrm{ic}$, and $\Delta \mathrm{ec}+\mathrm{ic}$ and the parental TCR: $\zeta$, all containing an intact $\mathrm{CD} 3 \zeta$ tm domain, showed no association with endogenous TCR chains (Fig. 3C and Supplementary Fig. 2). Notably, flow cytometry of TCR: $\zeta \Delta \mathrm{tm}+\mathrm{ic}$ and TCR: $\zeta \Delta \mathrm{ec}+\mathrm{tm}$ showed cells that were single positive for either TCR-V $\alpha 19$ or $-\mathrm{V} \beta 9$, respectively (Fig. 2A), indicating that $\mathrm{CD} 3 \zeta$ ec or ic domains do not completely prevent these TCRs from mis-pairing. Our finding that prevention of TCR mis-pairing, and consequently enhanced pairing between TCR $\alpha: \zeta$ and $-\beta: \zeta$, is governed by the CD3 $\zeta$ tm domain is consistent with a report by Rutledge and colleagues [38], in which the $\mathrm{CD} 3 \zeta$ tm domain was used to induce dimerization of monomeric proteins, such as the IL-2R $\alpha$ chain.
In the next series of experiments, we have transplanted a limited set of CD3 $\zeta$ tm amino acids onto TCR $\alpha$ and TCR $\beta$ with the intent to preserve surface expression and inability to TCR mis-pair and, at the same time, retain structural and spatial requirements to associate with endogenous CD3 chains (i.e., TCR:tm $\zeta 1$ and -2, (see Fig. 1)). This 'transplantation set' of amino acids was identified through 3D modeling and included the ones defined by Call and colleagues to be critically involved in $\mathrm{CD} 3 \zeta$ homodimerization (i.e., $\mathrm{C} 2$, D6, L9, Y12, T17 and F20) [39]. We observed that TCR:tm $\zeta$ heterodimers did not express at the cell surface (Fig. 2A). In fact, TCR $\alpha: \operatorname{tm} \zeta 1$ and -2 chains were not able to complex with TCR $\beta: \operatorname{tm} \zeta$ nor endogenous TCR $\beta$, and TCR $\beta: \operatorname{tm} \zeta$ showed moderate levels of surface expression through mispairing with the endogenous $\mathrm{TCR} \alpha$. These data suggest that TCR: $\zeta$ properties related to the presence of an intact $\mathrm{CD} 3 \zeta$ tm domain, such as enhanced cell surface expression and inability to associate with endogenous CD3 and TCR chains, cannot be attributed to a limited number of individual $\mathrm{CD} 3 \zeta$ tm amino acids.

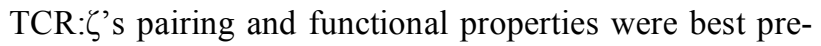
served in minimal TCR: $\zeta \Delta$ ec. This TCR variant retains high surface expression levels (Fig. 2), prevents pairing with endogenous CD3 and TCR chains (Fig. 3), binds pMHC (although binding is somewhat reduced when compared to

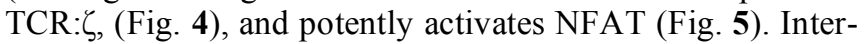
estingly, recent confocal microscopy studies demonstrated that minimal TCR: $\zeta \Delta \mathrm{ec}$ is able to form immunological synapses with similar sizes as those formed by parental TCR: $\zeta$ [20]. In minimal TCR: $\zeta \Delta \mathrm{ec}$ there is only a single artificial boundary, i.e., the one between TCR ec and CD3 $\zeta$ tm sequences, potentially preventing or diminishing humoral and/or cellular immunogenicity. Collectively, our observations warranted testing of minimal TCR: $\zeta \Delta \mathrm{ec}$ in primary human $\mathrm{T}$ cells. In bulk populations of human PBMC (nonsorted for either TCR or CD8 expression), we observed that the difference in surface expression between minimal TCR: $\zeta$ $\Delta \mathrm{ec}$ and parental TCR: $\zeta$ was more pronounced when compared to Jurkat T cells (Fig. 6A). Also, minimal TCR: $\zeta \Delta \mathrm{ec}$ showed lower pMHC binding and induced negligible CD107a mobilization to the cell surface when compared to TCR: $\zeta$ (Figs. 6B, C), which most likely was accounted for by the low level of surface expression. These observations, perhaps unexpectedly, were not different when using minimal TCR: $\zeta \Delta$ ec in an optimal vector, an optimal TCR cassette and with codon optimized $\mathrm{CD} 3 \zeta \mathrm{tm}+\mathrm{ic}$ domains. Apparently, the absence of the membrane-proximal $\mathrm{CD} 3 \zeta$ ec domain in minimal TCR: $\zeta \Delta \mathrm{ec}$ results in a stringent decrease in functional TCR expression. Interestingly, single chain (sc) Fv [40] and scTCR ([15], and data not shown) that do contain $\mathrm{CD} 3 \zeta$ ec coupled to either CD3 $\zeta \mathrm{tm}+\mathrm{ic}, \mathrm{CD} 4 \mathrm{tm}+$ $\operatorname{Fc}(\varepsilon) \mathrm{RI} \gamma$ ic, or $\operatorname{Fc}(\varepsilon) \mathrm{RI} \gamma \mathrm{tm}+\mathrm{ic}$, have indeed demonstrated significant surface expression in human T cells. In minimal TCR: $\zeta \Delta \mathrm{ec}$, six CD3 $\zeta$ ec amino acids (GDLDPK) were replaced by either TCR $\alpha$ (DVKLVEKSFETDTNLNFQNLS) or TCR $\beta$ (GFTSESYQQGVLSATILYE) ec amino acids (that cover the connecting-peptide motifs CPM $\alpha$ and CPM $\beta$, respectively). The CPM $\alpha$ and $C P M \beta$ are reported to interact with tetracysteine motifs in the membrane-proximal stalk regions of $\mathrm{CD} 3 \delta \varepsilon$ and $\mathrm{CD} 3 \gamma \varepsilon$ dimers, and as such contribute to $\mathrm{TCR} / \mathrm{CD} 3$ complex formation and $\mathrm{T}$ cell activation [41, 
42]. In addition, a defective or mutated CPM $\alpha$ is compromised with respect to its association with CD8, translation of antigen-specific stimulation into phosphorylation of Lck, Fyn, and ZAP70, and production of IL-2 [41, 43, 44]. We observed that the presence of $\mathrm{CPM} \alpha$ and $-\beta$ in minimal TCR: $\zeta \Delta$ ec appears not sufficient to induce TCR/CD3 complex formation (Figs. 3A, B), suggesting a more critical role for intact TCR tm for association with CD3 chains [45]. The extent to which the presence of CPM $\alpha$ and $-\beta$ in minimal TCR: $\zeta \Delta$ ec improved pMHC binding in T cells is difficult to assess due to poor expression levels in primary human $\mathrm{T}$ cells (Fig. 6A) and non-specificity of pMHC binding in $\mathrm{CD} 8 \alpha$-transduced Jurkat $\mathrm{T}$ cells (data not shown). Nevertheless, earlier findings proved that M1/A1-specific TCR: $\zeta$, not containing $\mathrm{CPM} \alpha$ or $-\beta$, were able to associate with and depend on CD $8 \alpha$ for ligand binding $[20,46]$, and may suggest a non-dominant role of CPM with respect to TCR:CD8 $\alpha$ association.

An alternative TCR format that is designed to address TCR mis-pairing, in addition to TCR: $\zeta$, is TCR:mu+cys. In TCR:mu+cys two separate strategies have been combined: murinization of the TCR-C domain and introduction of cysteine amino acids at structurally favorable positions to allow formation of an additional disulfide bridge, which together result in enhanced functional expression [24, 47] (reviewed in [11]). Our studies with M1/A1-specific TCR:mu+cys, taken along as a control TCR in the present paper, confirmed this notion to some extent. In Jurkat T cells, TCR:mu+cys, when compared to wt TCR, showed similar levels of surface expression and pMHC binding, and somewhat enhanced levels of antigen-specific NFAT activation (Figs. 2A, $\mathbf{4}$ and 5), whereas in primary human T cells, TCR:mu+cys showed enhanced levels of surface expression and similar levels of pMHC binding and antigen-specific CD107a mobilization to the cell surface (Fig. 6). However, we did observe that TCR:mu+cys mediates a significantly enhanced antigenspecific IFN $\gamma$ response (data not shown). These findings, although not fully in accordance with previous reports and potentially unique to the TCR-V regions of the M1/A1 TCR $[24,47]$, generally argue that TCR:mu+cys improved functional TCR expression. Expectedly, enhanced functional TCR expression was related to enhanced preferential pairing between the two modified TCR:mu+cys chains, which was suggested by the flow cytometric absence of single TCR $\alpha$ or TCR $\beta$ positive cells (Fig. 2A). Unexpectedly, however, flow cytometric FRET (Fig. 3B) and single TCR chain transductions (Supplementary Fig. 2) clearly demonstrated that TCR:mu+cys mis-paired with endogenous TCR chains to the same extent as wt TCR. Also in vivo, murine TCRs with a cysteine modification did not fully prevent the pathology related to TCR mis-pairing, i.e., TCR transfer-induced Graft versus Host disease [10]. We therefore propose that from a safety point of view, TCR: $\zeta$ but not TCR:mu+cys provides a better alternative to wt TCR.

In conclusion, our studies showed that $\mathrm{CD} 3 \zeta$ domains

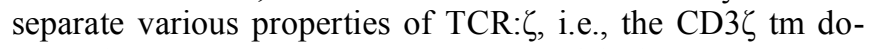
main determines surface expression and lack of association with endogenous CD3 and TCR chains, whereas CD3 $\zeta$ ic domain contributes to $T$ cell signaling. Functional expression of TCR: $\zeta$ in primary human T cells, however, required the complete rather than minimized content of $\mathrm{CD} 3 \zeta$.

\section{SUPPORTING INFORMATION}

Supporting information is available on the publishers Web site along with the published article.

\section{CONFLICT OF INTEREST}

None declared.

\section{ACKNOWLEDGEMENTS}

This work was supported by the European Union $6^{\text {th }}$ framework grant (018914) entitled: 'Adoptive engineered $\mathrm{T}$ cell targeting to activate cancer killing (ATTACK)'. Authors would like to acknowledge Prof. dr. Gert Vriend, Radboud University Nijmegen Medical Center, The Netherlands, for critically reviewing the manuscript.

\section{REFERENCES}

[1] Robbins PF, Morgan RA, Feldman SA, et al. Tumor Regression in Patients With Metastatic Synovial Cell Sarcoma and Melanoma Using Genetically Engineered Lymphocytes Reactive With NYESO-1. J Clin Oncol 2011; 29: 917-24.

[2] Parkhurst MR, Yang JC, Langan RC, et al. T cells targeting carcinoembryonic antigen can mediate regression of metastatic colorectal cancer but induce severe transient colitis. Mol Ther 2011; 19: 620-6.

[3] Morgan RA, Dudley ME, Wunderlich JR, et al. Cancer regression in patients after transfer of genetically engineered lymphocytes. Science 2006; 314: 126-9.

[4] Johnson LA, Morgan RA, Dudley ME, et al. Gene therapy with human and mouse T-cell receptors mediates cancer regression and targets normal tissues expressing cognate antigen. Blood 2009; 114: $535-46$

[5] Yee C, Thompson JA, Byrd D, et al. Adoptive T cell therapy using antigen-specific CD8+ $\mathrm{T}$ cell clones for the treatment of patients with metastatic melanoma: in vivo persistence, migration, and antitumor effect of transferred T cells. Proc Natl Acad Sci USA 2002; 99: 16168-73.

[6] Dudley ME, Wunderlich JR, Yang JC, et al. Adoptive cell transfer therapy following non-myeloablative but lymphodepleting chemotherapy for the treatment of patients with refractory metastatic melanoma. J Clin Oncol 2005; 23: 2346-57.

[7] Dudley ME, Wunderlich JR, Robbins PF, et al. Cancer regression and autoimmunity in patients after clonal repopulation with antitumor lymphocytes. Science 2002; 298: 850-4.

[8] Dudley ME, Gross CA, Langhan MM, et al. CD8+ enriched "young" tumor infiltrating lymphocytes can mediate regression of metastatic melanoma. Clin Cancer Res 2010; 16: 6122-31.

[9] Besser MJ, Shapira-Frommer R, Treves AJ, et al. Clinical responses in a phase II study using adoptive transfer of short-term cultured tumor infiltration lymphocytes in metastatic melanoma patients. Clin Cancer Res 2010; 16: 2646-55.

[10] Bendle GM, Linnemann C, Hooijkaas AI, et al. Lethal graftversus-host disease in mouse models of $\mathrm{T}$ cell receptor gene therapy. Nat Med 2010; 16: 565-70, 1p following 70.

[11] Govers C, Sebestyen Z, Coccoris M, Willemsen RA, Debets R. T cell receptor gene therapy: strategies for optimizing transgenic TCR pairing. Trends Mol Med 2010; 16: 77-87.

[12] Cohen CJ, Zhao Y, Zheng Z, Rosenberg SA, Morgan RA. Enhanced antitumor activity of murine-human hybrid T-cell receptor (TCR) in human lymphocytes is associated with improved pairing and TCR/CD3 stability. Cancer Res 2006; 66: 8878-86.

[13] Kuball J, Dossett ML, Wolfl M, et al. Facilitating matched pairing and expression of TCR chains introduced into human T cells. Blood 2007; 109: 2331-8.

[14] Voss RH, Willemsen RA, Kuball J, et al. Molecular design of the Calphabeta interface favors specific pairing of introduced TCRalphabeta in human T cells. J Immunol 2008; 180: 391-401.

[15] Willemsen RA, Weijtens ME, Ronteltap C, et al. Grafting primary human $\mathrm{T}$ lymphocytes with cancer-specific chimeric single chain and two chain TCR. Gene Ther 2000; 7: 1369-77. 
[16] Schaft N, Lankiewicz B, Drexhage J, et al. T cell re-targeting to EBV antigens following TCR gene transfer: CD28-containing receptors mediate enhanced antigen-specific IFNgamma production. Int Immunol 2006; 18: 591-601.

[17] Schaft N, Willemsen RA, de Vries J, et al. Peptide fine specificity of anti-glycoprotein $100 \mathrm{CTL}$ is preserved following transfer of engineered TCR alpha beta genes into primary human T lymphocytes. J Immunol 2003; 170: 2186-94.

[18] Sebestyen Z, Schooten E, Sals T, et al. Human TCR that incorporate CD3zeta induce highly preferred pairing between TCRalpha and beta chains following gene transfer. J Immunol 2008; 180: 7736-46.

[19] Yang W, Beaudoin EL, Lu L, et al. Chimeric immune receptors (CIRs) specific to JC virus for immunotherapy in progressive multifocal leukoencephalopathy (PML). Int Immunol 2007; 19: 1083-93.

[20] Roszik J, Sebestyen Z, Govers C, et al. T-cell synapse formation depends on antigen recognition but not $\mathrm{CD} 3$ interaction: studies with TCR:zeta, a candidate transgene for TCR gene therapy. Eur J Immunol 2011; 41: 1288-97.

[21] Sommermeyer D, Uckert W. Minimal amino acid exchange in human TCR constant regions fosters improved function of TCR gene-modified T cells. J Immunol 2010; 184: 6223-31.

[22] Van de Griend RJ, Van Krimpen BA, Bol SJ, Thompson A, Bolhuis RL. Rapid expansion of human cytotoxic $\mathrm{T}$ cell clones: growth promotion by a heat-labile serum component and by various types of feeder cells. J Immunol Methods 1984; 66: 285-98.

[23] Altman JD, Moss PA, Goulder PJ, et al. Phenotypic analysis of antigen-specific T lymphocytes. Science 1996; 274: 94-6.

[24] Cohen CJ, Li YF, El-Gamil M, Robbins PF, Rosenberg SA, Morgan RA. Enhanced antitumor activity of $\mathrm{T}$ cells engineered to express T-cell receptors with a second disulfide bond. Cancer Res 2007; 67: 3898-903

[25] Lamers $\mathrm{CH}$, Willemsen RA, van Elzakker P, van Krimpen BA, Gratama JW, Debets R. Phoenix-ampho outperforms PG13 as retroviral packaging cells to transduce human $\mathrm{T}$ cells with tumorspecific receptors: implications for clinical immunogene therapy of cancer. Cancer Gene Ther 2006; 13: 503-9.

[26] Szentesi G, Horvath G, Bori I, et al. Computer program for determining fluorescence resonance energy transfer efficiency from flow cytometric data on a cell-by-cell basis. Comput Methods Programs Biomed 2004; 75: 201-11.

[27] Aarnoudse CA, Kruse M, Konopitzky R, Brouwenstijn N, Schrier PI. TCR reconstitution in Jurkat reporter cells facilitates the identification of novel tumor antigens by cDNA expression cloning. Int J Cancer 2002; 99: 7-13.

[28] Schroten C, Kraaij R, Veldhoven JL, et al. T cell activation upon exposure to patient-derived tumor tissue: a functional assay to select patients for adoptive $\mathrm{T}$ cell therapy. J Immunol Methods 2010; 359: 11-20.

[29] Schaft N, Lankiewicz B, Gratama JW, Bolhuis RL, Debets R. Flexible and sensitive method to functionally validate tumorspecific receptors via activation of NFAT. J Immunol Methods 2003; $280: 13-24$

[30] Scholten KB, Kramer D, Kueter EW, et al. Codon modification of $\mathrm{T}$ cell receptors allows enhanced functional expression in transgenic human T cells. Clin Immunol 2006; 119: 135-45.

[31] Leisegang $M$, Engels $B$, Meyerhuber $P$, et al. Enhanced functionality of $\mathrm{T}$ cell receptor-redirected $\mathrm{T}$ cells is defined by the transgene cassette. J Mol Med 2008; 86: 573-83.

[32] Jorritsma A, Gomez-Eerland R, Dokter M, et al. Selecting highly affine and well-expressed TCRs for gene therapy of melanoma. Blood 2007; 110: 3564-72.
[33] Engels B, Cam H, Schuler T, et al. Retroviral vectors for high-level transgene expression in T lymphocytes. Hum Gene Ther 2003; 14: 1155-68.

[34] Lissina A, Ladell K, Skowera A, et al. Protein kinase inhibitors substantially improve the physical detection of T-cells with peptide-MHC tetramers. J Immunol Methods 2009; 340: 11-24.

[35] Luton F, Buferne M, Davoust J, Schmitt-Verhulst AM, Boyer C Evidence for protein tyrosine kinase involvement in ligand-induced $\mathrm{TCR} / \mathrm{CD} 3$ internalization and surface redistribution. J Immunol 1994; 153: 63-72.

[36] Zhao Y, Wang QJ, Yang S, et al. A herceptin-based chimeric antigen receptor with modified signaling domains leads to enhanced survival of transduced $\mathrm{T}$ lymphocytes and antitumor activity. J Immunol 2009; 183: 5563-74.

[37] Orlando L, Accomasso L, Circosta P, et al. TCR transfer induces TCR-mediated tonic inhibition of RAG genes in human T cells. Mol Immunol 2011; 48: 1369-76.

[38] Rutledge T, Cosson P, Manolios N, Bonifacino JS, Klausner RD Transmembrane helical interactions: zeta chain dimerization and functional association with the $\mathrm{T}$ cell antigen receptor. EMBO $\mathrm{J}$ 1992; 11: 3245-54.

[39] Call ME, Schnell JR, Xu C, Lutz RA, Chou JJ, Wucherpfennig $\mathrm{KW}$. The structure of the zetazeta transmembrane dimer reveals features essential for its assembly with the $\mathrm{T}$ cell receptor. Cell 2006; 127: 355-68.

[40] Weijtens ME, Willemsen RA, Hart EH, Bolhuis RL. A retroviral vector system 'STITCH' in combination with an optimized single chain antibody chimeric receptor gene structure allows efficient gene transduction and expression in human T lymphocytes. Gene Ther 1998; 5: 1195-203.

[41] Backstrom BT, Milia E, Peter A, Jaureguiberry B, Baldari CT, Palmer E. A motif within the T cell receptor alpha chain constant region connecting peptide domain controls antigen responsiveness. Immunity 1996; 5: 437-47.

[42] $\mathrm{Xu} \mathrm{C}$, Call ME, Wucherpfennig KW. A membrane-proximal tetracysteine motif contributes to assembly of CD3deltaepsilon and CD3gammaepsilon dimers with the T cell receptor. J Biol Chem 2006; 281: 36977-84.

[43] Mallaun M, Naeher D, Daniels MA, et al. The T cell receptor's alpha-chain connecting peptide motif promotes close approximation of the CD8 coreceptor allowing efficient signal initiation. J Immunol 2008; 180: 8211-21.

[44] Ulivieri C, Peter A, Orsini E, Palmer E, Baldari CT. Defective signaling to Fyn by a $\mathrm{T}$ cell antigen receptor lacking the alpha -chain connecting peptide motif. J Biol Chem 2001; 276: 3574-80

[45] Call ME, Pyrdol J, Wiedmann M, Wucherpfennig KW. The organizing principle in the formation of the $\mathrm{T}$ cell receptor-CD3 complex. Cell 2002; 111: 967-79.

[46] Willemsen R, Ronteltap C, Heuveling M, Debets R, Bolhuis R. Redirecting human $\mathrm{CD} 4+\mathrm{T}$ lymphocytes to the MHC class Irestricted melanoma antigen MAGE-A1 by TCR alphabeta gene transfer requires CD8alpha. Gene Ther 2005; 12: 140-6.

[47] Thomas S, Xue SA, Cesco-Gaspere M, et al. Targeting the Wilms tumor antigen 1 by TCR gene transfer: TCR variants improve tetramer binding but not the function of gene modified human $\mathrm{T}$ cells. J Immunol 2007; 179: 5803-10.

[48] Romero P, Pannetier C, Herman J, Jongeneel CV, Cerottini JC, Coulie PG. Multiple specificities in the repertoire of a melanoma patient's cytolytic $\mathrm{T}$ lymphocytes directed against tumor antigen MAGE-1.A1. J Exp Med 1995; 182: 1019-28. 